\section{De la diversité des cellules dendritiques humaines}

Gaëlle Breton
Laboratoire d'immunologie moléculaire, The Rockefeller University, 1230 York Avenue, Box 220, New York, Ny 10065, દ́tats-Unis. gbreton@mail.rockefeller.edu

dans le sang et dans les amygdales. II peut donc migrer de la moelle osseuse vers les organes lymphoïdes secondaires par la circulation sanguine.

Une même origine pour toutes

les cellules dendritiques

conventionnelles?

L'origine de la diversité fonctionnelle des cellules dendritiques n'est actuellement pas identifiée. Les cellules dendritiques $\mathrm{BDCA}-\mathrm{I}^{+}$et $\mathrm{BDCA}-3^{+}$se développentelles indépendamment ou ont-elles une origine commune, avant d'acquérir des fonctions spécifiques selon le milieu dans lequel elles se trouvent? Les pré-cDC sont à l'origine des deux sous-types de cellules dendritiques conventionnelles. Nous avons cependant observé in vitro qu'une cellule pré-cDC unique pouvait n'être à l'origine que de cellules dendritiques $\mathrm{BDCA}-\mathrm{I}^{+}$ou de cellules BDCA $-3^{+}$, mais très rarement des deux sous-types de cellules [6]. Chez la souris, les cellules pré-cDC contiennent en fait deux précurseurs qui sont déjà engagés dans la différenciation de l'une ou l'autre des sous-populations de cellules dendritiques $[8,9]$. Chez l'homme, les précDC pourraient donc être également hétérogènes. Des marqueurs spécifiques des différentes populations cellulaires semblent donc nécessaires afin d'isoler le précurseur à l'origine de chacune des sous-populations de cellules dendritiques conventionnelles.

Deux précurseurs à l'origine des cellules dendritiques $\mathrm{BDCA}-\mathrm{1}^{+}$et $\mathrm{BDCA}-3^{+}$

Afin d'étudier l'hétérogénéité des progéniteurs pré-cDC chez l'homme et ainsi d'identifier les précurseurs des cellules 


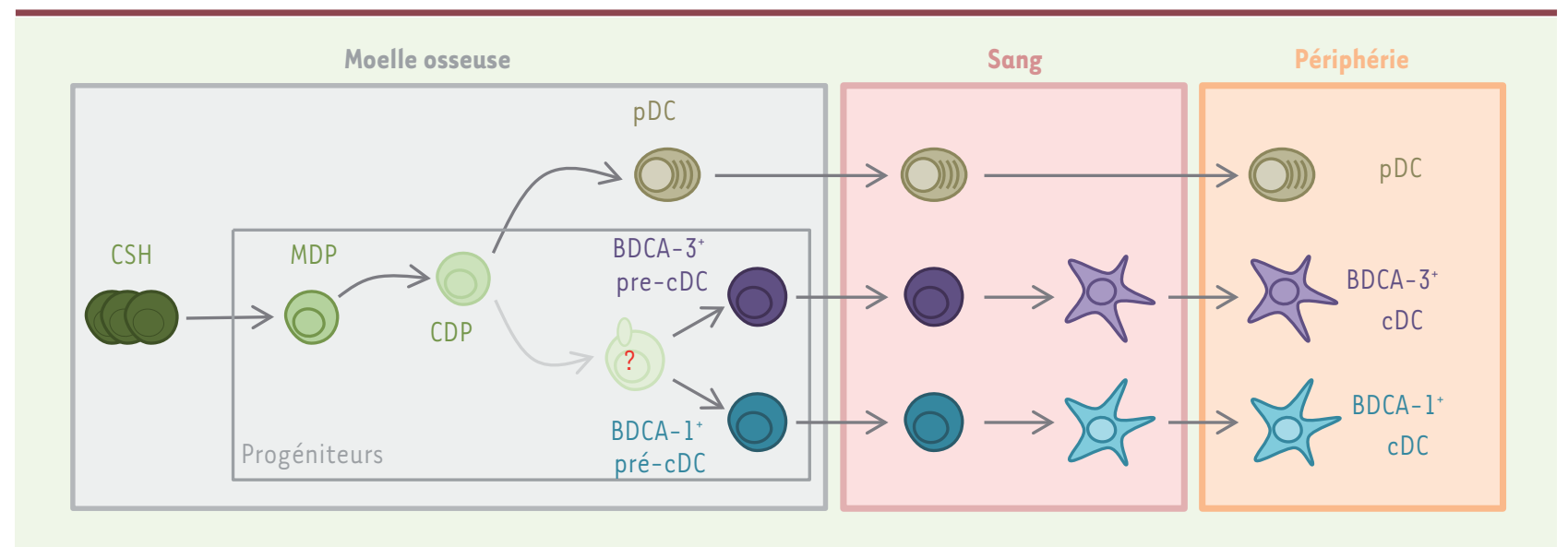

Figure 1. Développement des cellules dendritiques humaines. Trois sous-populations de cellules dendritiques ont été décrites chez l'homme : les cellules dendritiques plasmacytoïdes ( $p D C$ ), et deux populations de cellules dendritiques conventionnelles ( $c D C$ ) que l'on différencie par l'expression de BDCA-1 (blood dendritic cell antigen-1) ou de BDCA-3. Leur développement débute dans la moelle osseuse. Le progéniteur MDP (monocyte and dendritic cell progenitor), commun aux monocytes et aux cellules dendritiques, est à l'origine du précurseur commun aux trois sous-populations de cellules dendritiques, le CDP (common DC progenitor). Le CDP mature en pDC et est à l'origine de deux précurseurs immédiats des cellules dendritiques conventionnelles appelé BDCA $-1^{+}$pré-cDC (conventional dendritic cell precursor) et BDCA-3 $3^{+}$pré-cDC. Ces précurseurs migrent de la moelle osseuse vers les organes lymphoïdes secondaires via la circulation sanguine et sont à l'origine des deux sous-populations principales de cellules dendritiques conventionnelles, nommées BDCA $-1^{+} \mathrm{CDC}$ et $\mathrm{BDCA}-3^{+} \mathrm{cDC}$. L'existence d'un précurseur commun aux cellules dendritiques $B D C A-1^{+}$et $B D C A-3^{+} n^{\prime}$ est pas connu. $C S H$ : cellules souches hématopoiétiques.

dendritiques $\mathrm{BDCA}-\mathrm{I}^{+}$et $\mathrm{BDCA}-3^{+}$, nous avons examiné le transcriptome au niveau de la cellule, d'environ 450 cellules pré-cDC isolées de sang de cordon ou de sang périphérique humains [10]. Cette méthode permet une analyse détaillée de l'expression génique au niveau de la cellule individuelle. Elle donne également des indications quant à la nature et à l'étendue de l'hétérogénéité d'une population cellulaire. Par cette analyse, nous avons montré que les pré-cDC constituent en réalité un groupe de cellules hétérogène regroupant des cellules qui expriment les marqueurs caractéristiques des cellules dendritiques BDCA- $1^{+}$(Clecl0A [C-type lectin domain family 10], عTS2 [erythroblastosis virus $\varepsilon 26$ oncogene homolog-2], ZEB2 [zinc finger $\varepsilon$-box binding bomeobox 2]) ou les marqueurs des cellules dendritiques BDCA-3+ (Clec9A, ID02 [indoleamine 2,3-dioxygenase 2], BATF3 [basic leucine zipper ATF-like transcription factor 3]). Afin de distinguer ces deux sous-types de précurseurs au sein de la population pré-
cDC étudiée, nous avons recherché des marqueurs spécifiquement exprimés par l'une ou l'autre des sous-populations de cellules dendritiques différenciées. Nous avons montré que les deux types de précurseurs peuvent en fait être différenciés selon leur expression de CD172a (ou SIRPa [signal protein regulatory $\alpha]$ ), les pré-cDC exprimant ce marqueur étant les précurseurs des cellules $B D C A-1^{+}$, les pré-cDC CD172a étant à l'origine des cellules BDCA-3+ (Figure I). Chaque sous-population de pré-cDC est présente dans la moelle osseuse mais également dans le sang périphérique et dans le sang de cordon. Elles prolifèrent et présentent un phénotype plus immature que les cellules dendritiques différenciées. L'analyse transcriptomique réalisée sur les précurseurs exprimant CD172a (les BDCA- $1^{+}$pré-cDC) et ceux ne l'exprimant pas (les $B D C A-3^{+}$préCDC) a montré qu'ils représentaient deux populations distinctes qui expriment des facteurs de transcription spécifiques des lignées BDCA-1 ${ }^{+}$ou BDCA-3+ $3^{+}$. L'injection de Flt3I (FMS-like tyrosine kinase 3 ligand), hématopoïétine nécessaire pour le développement des cellules dendritiques, permet d'augmenter le nombre de cellules des deux sous-types de précurseurs dans le sang de volontaires et cela de façon similaire à l'augmentation observée pour les trois populations de cellules dendritiques matures. Notre étude ne nous a cependant pas permis d'identifier un précurseur commun pour les cellules dendritiques BDCA- $1^{+}$et les cellules $\mathrm{BDCA}-3^{+}$. Son existence reste néanmoins possible.

\section{Conclusion et perspectives}

L'identification de deux précurseurs qui se différencient spécifiquement en cellules dendritiques $B D C A-1^{+}$ou en cellules dendritiques $B D C A-3^{+}$constitue une étape importante dans notre compréhension des cellules dendritiques. Cependant, le contrôle du développement de ces deux populations dans un contexte non-pathologique reste incompris. Savoir si différentes conditions d'inflammation ou d'infection favorisent le développement d'une 
population au détriment de l'autre reste également à définir. Comprendre comment ces populations sont régulées est primordial afin de déterminer le rôle de ces cellules dans les différentes pathologies dans lesquelles elles interviennent, mais également pour le développement de nouvelles approches thérapeutiques. $\diamond$

Origin of human dendritic cell diversity

\section{LIENS D'INTÉRÊT}

Les auteurs déclarent n'avoir aucun lien d'intérêt concernant les données publiées dans cet article.

\section{RÉFÉRENCES}

1. Collin M, McGovern N, Haniffa M. Human dendritic cell subsets. Immunology $2013 ; 140: 22-30$.

2. Melissa Swiecki, Marco Colonna. The multifaceted biology of plasmacytoid dendritic cells. Nat Rev Immunol $2015 ; 15: 471-85$.

3. Schlitzer A, McGovern N, Teo P, et al. IRF4 transcription factor-dependent CDIlb+ dendritic cells in human and mouse control mucosal IL-17 cytokine responses. Immunity $2013 ; 38: 970-83$.

4. Villadangos JA, Shortman K. Found in translation: the human equivalent of mouse CD8+ dendritic cells. J Exp Med 2010 ; 207 : 1131-4

5. Lee J, Breton G, Oliveira Ty, et al. Restricted dendritic cell and monocyte progenitors in human cord blood and bone marrow. J Exp Med 2015 ; 212 : 385-99.

6. Breton G, Lee J, Zhou YJ, et al. Circulating precursors of human $\mathrm{CDIC}^{+}$and $\mathrm{CDI}_{41} \mathrm{l}^{+}$dendritic cells. J Exp Med 2015 ; $212: 401-13$
7. Breton G. Origine et développement des cellules dendritiques humaines. Med Sci (Paris) 2015 ; 31 : 725-7.

8. Schlitzer $A$, Sivakamasundari V, Chen J, et al. Identification of $\mathrm{CDCl}^{-}$and $\mathrm{CDC2}^{-}$committed $\mathrm{DC}$ progenitors reveals early lineage priming at the common DC progenitor stage in the bone marrow. Nat Immunol $2015 ; 16: 718-28$.

9. Grajales-Reyes GE, Iwata A, Albring J, et al. Batf3 maintains autoactivation of Irf8 for commitment of a CD $8 \alpha^{+}$conventional DC clonogenic progenitor. Nat Immunol 2015 ; 16 : 708-17.

10. Breton G, Zheng S, Valieris R, Tojal da Silva I, Satija R, Nussenzweig MC. Human dendritic cells (DCs) are derived from distinct circulating precursors that are precommitted to become $\mathrm{CD} 1 \mathrm{c}^{+}$or $\mathrm{CD} 14 \mathrm{1}^{+} \mathrm{DC}$. J Exp Med $2016 ; 213: 2861-70$.

\section{NOUVELle}

\section{Motivation et apathie parkinsoniennes}

\section{Rôle des récepteurs dopaminergiques $\mathbf{R D}_{\mathbf{3}}$}

Mathieu Favier ${ }^{1,2}$, Carole Carcenac ${ }^{1,2}$, Marc Savasta $^{1-3}$,

Sébastien Carnicella ${ }^{1,2}$
${ }^{1}$ Inserm U1216, physiopathologie de la motivation, site Santé La Tronche, chemin Fortuné Ferrini, BP 170, 38042 Grenoble, France.

${ }^{2}$ Université Grenoble Alpes, Grenoble institut des neurosciences (GIN), 38000 Grenoble, France.

${ }^{3}$ Centre Hospitalier Universitaire de Grenoble, BP 217, 38043 Grenoble, France.

sebastien.carnicella@inserm.fr
> Au-delà des symptômes moteurs qui la caractérisent avec, notamment, la triade akinésie-rigidité-tremblement, qui sont induits par la dégénérescence des neurones dopaminergiques de la substance noire pars compacta (SNc), la maladie de Parkinson (MP) a récemment été identifiée comme une maladie neuropsychiatrique à part entière $[1$, 2]. En effet, elle s'accompagne très fréquemment de troubles du comportement - notamment l'apathie, la dépression et l'anxiété - qui peuvent apparaître quelques mois, voire quelques années, avant la survenue des symptômes moteurs [1, 2]. Ces troubles dits «non moteurs», ont une étiologie complexe qui reste peu comprise, ce qui rend leur prise en charge délicate. Parmi ces troubles, l'apathie est I'un des symptômes les plus fréquents de la MP. Elle contribue, au moins autant que les troubles moteurs, à altérer la qualité de vie des patients et à une morbidité élevée $[1,2]$. L'apathie est définie comme un manque de motivation ou une réduction des comportements dirigés vers un but, qui résultent cliniquement d'un déficit des comportements auto-initiés, volontaires et déterminés [1, 3]. Une étude menée par Schmidt et ses collaborateurs a montré que les patients apathiques souffrent d'une incapacité à produire un effort et à mettre en place une action adaptée permettant d'obtenir une récompense attendue, bien que la perception de la valeur de cette récompense reste inchangée [4]. Ceci suppose que l'apathie est spécifiquement liée à des dysfonctionnements sévères des processus préparatoires motiva- tionnels. Ces symptômes apathiques sont plus particulièrement observés chez les patients parkinsoniens non traités. Toutefois, ils sont également retrouvés chez les patients traités pour lesquels la médication (ayant pour but de rétablir l'activité déficitaire de la transmission dopaminergique, comme la L-dopa ou les agonistes dopaminergiques) est fortement diminuée, par exemple à la suite de la mise en place d'une stimulation cérébrale profonde. Ces symptômes peuvent être toutefois améliorés, voire même abolis, lorsque les traitements dopaminergiques sont adaptés au patient [1]. L'apathie parkinsonienne semble donc dépendre de l'état fonctionnel de la neurotransmission dopaminergique, suggérant un rôle critique de ce neurotransmetteur dans sa physiopathologie [1]. 